\title{
Quantitative target analysis and kinetic profiling of acyl-CoAs reveal the rate-limiting step in cyanobacterial 1-butanol production
}

\author{
Shingo Noguchi ${ }^{1} \cdot$ Sastia P. Putri $^{1} \cdot$ Ethan I. Lan $^{2} \cdot$ Walter A. Laviña ${ }^{1}$. \\ Yudai Dempo $^{1} \cdot$ Takeshi Bamba $^{1} \cdot$ James C. Liao $^{2} \cdot$ Eiichiro Fukusaki $^{1}$
}

Received: 21 August 2015/Accepted: 13 November 2015/Published online: 4 January 2016

(c) The Author(s) 2016. This article is published with open access at Springerlink.com

\begin{abstract}
Cyanobacterial 1-butanol production is an important model system for direct conversion of $\mathrm{CO}_{2}$ to fuels and chemicals. Metabolically-engineered cyanobacteria introduced with a heterologous Coenzyme A (CoA)dependent pathway modified from Clostridium species can convert atmospheric $\mathrm{CO}_{2}$ into 1-butanol. Efforts to optimize the 1-butanol pathway in Synechococcus elongatus PCC 7942 have focused on the improvement of the CoAdependent pathway thus, probing the in vivo metabolic state of the CoA-dependent pathway is essential for identifying its limiting steps. In this study, we performed quantitative target analysis and kinetic profiling of acylCoAs in the CoA-dependent pathway by reversed phase ion-pair liquid chromatography-triple quadrupole mass spectrometry. Using ${ }^{13} \mathrm{C}$-labelled cyanobacterial cell extract as internal standard, measurement of the intracellular concentration of acyl-CoAs revealed that the reductive reaction of butanoyl-CoA to butanal is a possible ratelimiting step. In addition, improvement of the butanoylCoA to butanal reaction resulted in an increased rate of acetyl-CoA synthesis by possibly compensating for the limitation of free CoA species. We inferred that the
\end{abstract}

Electronic supplementary material The online version of this article (doi:10.1007/s11306-015-0940-2) contains supplementary material, which is available to authorized users.

Eiichiro Fukusaki

fukusaki@bio.eng.osaka-u.ac.jp

1 Department of Biotechnology, Graduate School of Engineering, Osaka University, 2-1Yamadaoka, Suita, Osaka 565-0871, Japan

2 Department of Chemical and Biomolecular Engineering, University of California, Los Angeles, 5531 Boelter Hall, 420 Westwood Plaza, Los Angeles, CA 90095, USA efficient recycling of free CoA played a key role in enhancing the conversion of pyruvate to acetyl-CoA.

Keywords Cyanobacteria $\cdot$ 1-Butanol $\cdot$ Acyl-CoAs . Quantitative target analysis - Kinetic profiling - Liquid chromatography-mass spectrometry (LC-MS)

\section{Introduction}

Cyanobacterial biofuel production is considered as an attractive approach for providing sustainable energy resources due to its capability to utilize solar energy and $\mathrm{CO}_{2}$ as sole energy and carbon sources, respectively (Machado and Atsumi 2012; Jin et al. 2014). So far, production of various biofuels such as ethanol (Gao et al. 2012; Deng and Coleman 1999; Dexter and Fu 2009), 1-butanol (Lan and Liao 2011, 2012; Lan et al. 2013), isobutanol (Atsumi et al. 2009; Varman et al. 2012), 2,3butanediol (Oliver et al. 2013), fatty acid (Liua et al. 2011), alkanes (Wang et al. 2013) and isoprene (Lindberg et al. 2010) have been achieved via engineered bioprocess in cyanobacteria.

1-Butanol is a promising gasoline replacement compared to the more commonly used ethanol due to several advantages. Specifically, 1-butanol is less corrosive and has a higher energy density than ethanol (Dürre 2007). Previously, we were able to successfully engineer the cyanobacteria Synechococcus elongates PCC 7942 to produce 1-butanol under anoxic and dark conditions via a modified CoA-dependent pathway (Lan and Liao 2011) based on the acetone-butanol-ethanol fermentation in Clostridium species (Gheshlaghi et al. 2009). In a stepwise process, we enabled cyanobacterial 1-butanol production under photosynthetic condition by introducing ATP as an 
alternative driving force for acetoacetyl-CoA synthesis, which is the first committed step in the 1-butanol pathway (Lan and Liao 2012). Furthermore, 1-butanol productivity was dramatically improved under photosynthetic condition by substituting the oxygen-sensitive butanal dehydrogenase with an oxygen-tolerant CoA-acylating propionaldehyde dehydrogenase (Lan et al. 2013). However, the maximum titer in cyanobacteria is still low compared to the 1-butanol-producing Escherichia coli with the heterologous CoA-dependent pathway; the 1-butanol titer is $317 \mathrm{mg} / \mathrm{L}$ from $\mathrm{CO}_{2}$ in 12 days using Synechococcus elongatus and $30 \mathrm{~g} / \mathrm{L}$ from glucose in 7 days using Escherichia coli (Lan et al. 2013; Shen et al. 2011). Although the improvement of 1-butanol productivity in cyanobacteria has been made by focusing on the CoA-dependent 1-butanol biosynthesis pathway, there is no information about in vivo metabolic state of the CoA-dependent pathway in cyanobacteria. Therefore, intracellular metabolic profiling of the intermediates in the CoA-dependent pathway is expected to be essential for gaining clues for further optimization of the 1-butanol biosynthesis pathway.

To the best of our knowledge, there is no report on the investigation of the in vivo metabolic state of 1-butanolproducing cyanobacteria in spite of the attention given to photosynthetic 1-butanol production system. To tackle this problem, we performed targeted quantitative analysis and kinetic profiling of acyl-CoAs in the modified CoA-dependent pathway by using reversed phase ion-pair liquid chromatography-triple quadrupole mass spectrometry (RPIP-LC)/QqQ-MS. This study aimed to gain insights into further CoA-dependent pathway optimization for the improvement of 1-butanol productivity in Synechococcus elongatus. Our results validated at the metabolite level that ATP-driven malonyl-CoA-mediated pathway functioned as intended and indicated a possible rate-limiting reaction in the pathway. Moreover, this study revealed an unexpected increase in the acetyl-CoA synthesis rate due to the introduction of the oxygen-tolerant aldehyde dehydrogenase and explained the possible reason for the enhanced conversion of pyruvate to acetyl-CoA. The results of this study will be helpful in improving productivity of 1-butanol and other chemicals in cyanobacteria via the modification of the CoA-dependent pathway.

\section{Materials and methods}

\subsection{Cyanobacterial strains, plasmids and oligonucleotides used}

Synechococcus elongatus strains used in this study are listed in Table 1. Plasmid pEL256 was constructed by excluding genes $p d u P$ and $y q h D$ from plasmid pSR3 (Lan et al. 2013). Using pSR3 as template, fragments 1 and 2 were amplified by PCR with primer pairs EL-pEL256-P1F/EL-pEL256-P1-R and EL-pEL256-P1-F/ EL-pEL256$\mathrm{P} 2-\mathrm{R}$, respectively. The two fragments were then ligated using the Gibson isothermal DNA assembly method (Gibson et al. 2009). Strain BUOH-SE w/o pduP was constructed by transforming strain EL9 with plasmid pEL256.

\subsection{Culture medium and growth conditions}

The culture medium and conditions used in this study were based on Lan et al. (2013) with minor modifications. All cyanobacterial strains were grown on modified BG-11 plates $\left(1.5 \mathrm{~g} / \mathrm{L} \mathrm{NaNO} 3,0.0272 \mathrm{~g} / \mathrm{L} \mathrm{CaCl}{ }_{2} \cdot 2 \mathrm{H}_{2} \mathrm{O}, 0.012 \mathrm{~g} / \mathrm{L}\right.$ ferric ammonium citrate, $0.001 \mathrm{~g} / \mathrm{L} \mathrm{Na} 2$ EDTA, $0.040 \mathrm{~g} / \mathrm{L}$ $\mathrm{K}_{2} \mathrm{HPO}_{4}, 0.0361 \mathrm{~g} / \mathrm{L} \mathrm{MgSO}{ }_{4} \cdot 7 \mathrm{H}_{2} \mathrm{O}, 0.020 \mathrm{~g} / \mathrm{L} \mathrm{Na}_{2} \mathrm{CO}_{3}, \times$ 1000 trace mineral, $1.43 \mathrm{~g} \mathrm{H}_{3} \mathrm{BO}_{3}, 0.905 \mathrm{~g} \mathrm{MnCl}_{2} \cdot 4 \mathrm{H}_{2} \mathrm{O}$, $0.111 \mathrm{~g} \mathrm{ZnSO}_{4} \cdot 7 \mathrm{H}_{2} \mathrm{O}, 0.195 \mathrm{~g} \mathrm{Na}_{2} \mathrm{MoO}_{4} \cdot 2 \mathrm{H}_{2} \mathrm{O}, 0.0395 \mathrm{~g}$ $\mathrm{CuSO}_{4} \cdot 5 \mathrm{H}_{2} \mathrm{O}, \quad 0.0245 \mathrm{~g} \quad \mathrm{Co}\left(\mathrm{NO}_{3}\right)_{2} \cdot 6 \mathrm{H}_{2} \mathrm{O}, \quad 0.00882 \mathrm{~g} / \mathrm{L}$ sodium citrate dihydrate) agar $(1.5 \% \mathrm{w} / \mathrm{v})$. The strains were cultured in $50 \mathrm{~mL}$ of BG-11 medium containing $50 \mathrm{mM} \mathrm{NaHCO}$ and $20 \mathrm{mM}$ HEPES-KOH (pH 7.5) in $300 \mathrm{~mL}$ screw cap flasks. In the case of mutants, $20 \mathrm{mg} / \mathrm{L}$ spectinomycin and $10 \mathrm{mg} / \mathrm{L}$ kanamycin were added. Three different flasks were prepared for each strain as biological replicates. Cultures were grown with continuous shaking at $30{ }^{\circ} \mathrm{C}$ under $50 \mu \mathrm{mol} / \mathrm{s} / \mathrm{m}^{2}$ light condition. The initial cell density of culture was set at $\mathrm{OD}_{730}=0.04$. Induction of the growing culture with IPTG (1 $\mathrm{mM}$ final concentration) was done at cell density $\mathrm{OD}_{730}$ equal to $0.3-0.4$. The $\mathrm{pH}$ was adjusted to eight everyday using $10 \mathrm{M} \mathrm{HCl}$. After 1 day of IPTG induction, $5 \mathrm{~mL}$ of the culture was removed from the flask and $5 \mathrm{~mL}$ of fresh BG-11 containing $500 \mathrm{mM} \mathrm{NaHCO} 3$ and IPTG were added back to the culture.

\subsection{Sampling and extraction procedure}

Cells were harvested by fast filtration using $0.2 \mu \mathrm{m}$ pore size Omnipore filter disks (Millipore, USA) and subsequently washed with pre-cooled $70 \mathrm{mM}$ ammonium bicarbonate. The filter papers containing the cells were immediately placed in an aluminum cylinder pre-chilled with liquid nitrogen for immediate quenching of cyanobacterial metabolic activity. The quenching procedure was performed in less than $30 \mathrm{~s}$. The filter papers were stored in $15 \mathrm{~mL}$ centrifuge tubes at $-80{ }^{\circ} \mathrm{C}$ until extraction.

For quantitative target analysis, culture broth equivalent to $5 \mathrm{mg}$ dry cell weight was harvested after 3 days of IPTG induction. Intracellular metabolites were extracted using $2 \mathrm{~mL}$ of pre-chilled $80 \%$ methanol with $100 \mu \mathrm{L}$ of ${ }^{13} \mathrm{C}$ - 
Table 1 Strains, plasmids and oligonucleotides used

\begin{tabular}{|c|c|c|c|c|}
\hline Strains & \multicolumn{2}{|c|}{ Important Genotype } & $\begin{array}{l}\text { Reported } \\
\text { 1-butanol } \\
\text { production } \\
(\mathrm{mg} / \mathrm{L})\end{array}$ & Source \\
\hline PCC 7942 & \multicolumn{4}{|c|}{ Wild-type Synechococcus elongatus PCC 7942} \\
\hline EL9 & \multicolumn{3}{|c|}{$\mathrm{P}_{\text {Trc }}::$ His-tagged $T$. denticola ter integrated at NSI in PCC 7942 genome } & $\begin{array}{l}\text { Lan and Liao } \\
2011\end{array}$ \\
\hline EL14 & \multicolumn{2}{|c|}{$\begin{array}{l}\mathrm{P}_{\text {Trc }}:: \text { His-tagged } T \text {. denticola ter integrated at NSI and } \mathrm{P}_{\mathrm{LlacO} 1}:: \text { atoB, adhE2,crt, hbd } \\
\text { integrated at NSII in PCC } 7942 \text { genome }\end{array}$} & $<2$ & $\begin{array}{l}\text { Lan and Liao } \\
\text { 2011, } 2012\end{array}$ \\
\hline EL20 & \multicolumn{2}{|c|}{$\begin{array}{l}\mathrm{P}_{\text {Trce: }}: \text { His-tagged } T \text {. denticola ter integrated at NSI and } \mathrm{P}_{\mathrm{LlacO} 1}:: n p h T 7, \text { adhE2, crt, hbd } \\
\text { integrated at NSII in PCC } 7942 \text { genome }\end{array}$} & 6.4 & $\begin{array}{l}\text { Lan and Liao } \\
2012\end{array}$ \\
\hline EL22 & \multicolumn{2}{|c|}{$\begin{array}{l}\mathrm{P}_{\text {Trc }}:: \text { His-tagged } T \text {. denticola ter integrated at NSI and } \mathrm{P}_{\mathrm{LlacO} 1}:: n p h T 7, \text { bldh, yqhD, phaJ, } \\
\text { phaB integrated at NSII in PCC } 7942 \text { genome }\end{array}$} & 29.9 & $\begin{array}{l}\text { Lan and Liao } \\
2012\end{array}$ \\
\hline BUOH-SE & \multicolumn{2}{|c|}{$\begin{array}{l}\mathrm{P}_{\text {Trc }}: \text { His-tagged } T \text {. denticola ter integrated at NSI and } \mathrm{P}_{\mathrm{LlacO} 1}:: n p h T 7, p d u P \_S . \text { ent, yqhD, } \\
\text { phaJ, phaB integrated at NSII }\end{array}$} & 317 & Lan et al. 2013 \\
\hline $\begin{array}{l}\text { BUOH-SE } \\
\text { w/o pduP }\end{array}$ & \multicolumn{3}{|c|}{$\begin{array}{l}\mathrm{P}_{\text {Trc }}:: \text { His-tagged } T . \text { denticola ter integrated at NSI and } \mathrm{P}_{\mathrm{LlacO} 1}:: n p h T 7, \text { phaJ, phaB integrated } \\
\text { at NSII }\end{array}$} & This study \\
\hline Plasmids & \multicolumn{2}{|c|}{ Genotype } & $\begin{array}{l}\text { Reported 1-butanol } \\
\text { production (mg/L) }\end{array}$ & Source \\
\hline pSR3 & \multirow{2}{*}{\multicolumn{2}{|c|}{$\begin{array}{l}\text { Kan }^{\mathrm{R}} \text {; NSII targeting; } \mathrm{P}_{\mathrm{LlacO} 1}:: n p h T 7, \text { pduP_S. ent, phaJ, phaB } \\
\mathrm{Kan}^{\mathrm{R}} \text {; NSII targeting; } \mathrm{P}_{\mathrm{LlacO} 1}:: n p h T 7, \text { phaJ, phaB }\end{array}$}} & & Lan et al. 2013 \\
\hline pEL256 & & & & This study \\
\hline Primers & & $\begin{array}{l}\text { Reported 1-b } \\
\text { production }(1\end{array}$ & $\begin{array}{l}\text { Reported 1-butanol } \\
\text { production }(\mathrm{mg} / \mathrm{L})\end{array}$ & Source \\
\hline \multicolumn{2}{|c|}{ EL-pEL256-P1-F } & AGGAGATATACCATGTCTGCGCAATCTCTC & & This study \\
\hline \multicolumn{2}{|c|}{ EL-pEL256-P1-R } & CTAGATCTCGCAGCGTAAAGCCG & & This study \\
\hline \multicolumn{2}{|c|}{ EL-pEL256-P1-F } & CGGCTTTACGCTGCGAGATCTAG & & This study \\
\hline \multicolumn{2}{|c|}{ EL-pEL256-P2-R } & CATGGTATATCTCCTTTACCACTCGATCAGC & & This study \\
\hline
\end{tabular}

Oligonucletide primer sequence is from $5^{\prime}$ to $3^{\prime}$. Reported 1-butanol production values are according to the corresponding source publications. For EL14, EL20 and EL22 each data was taken at 18 days since IPTG induction under light and aerobic condition (Lan and Liao 2012). For BUOH-SE the data was taken at 12 days since IPTG induction under light and aerobic condition (Lan et al. 2013)

$\operatorname{Ter}$ (T. denticola) trans-2-enoyl-CoA reductase, atoB (E. coli) acetyl-CoA acetyltransferase, adhE (C. acetobutylicum) bifunctional aldehyde/ alcohol dehydrogenase, crrt (C. acetobutylicum) hbd (C. acetobutylicum), nphT7 (Streptomyces sp. Strain CL190) acetoacetyl-CoA synthase, bldh (C. saccharoperbutylacetonicum) CoA-acylating butanal dehydrogenase, yqhD (E. coli) NADPH-dependent alcohol dehydrogenase, phaJ (A. caviae) $(R)$-specific crotonase, phaB (R. eutropha) acetoacetyl-CoA reductase, pduP (S. enterica) CoA-acylating propionaldehyde dehydrogenase, $\operatorname{Kan}^{R}$ kanamycin resistance

internal standard followed by vortexing for $30 \mathrm{~s}$ and sonication for $20 \mathrm{~s}$. After precipitating the cells by centrifugation at $10,000 \times g$ for $5 \mathrm{~min}$ at $4{ }^{\circ} \mathrm{C}$, two separate $2 \mathrm{~mL}$ microfuge tubes were each dispensed with $800 \mu \mathrm{L}$ of the resulting supernatant. Subsequently, $640 \mu \mathrm{L}$ of chloroform and $480 \mu \mathrm{L}$ of ultrapure water were added into each tube before vortexing for $30 \mathrm{~s}$. The samples were centrifuged at $16,000 \times g$ for $5 \mathrm{~min}$ at $4{ }^{\circ} \mathrm{C}$ and $800 \mu \mathrm{L}$ of the resulting polar phase was collected and filtered using a $0.20 \mu \mathrm{m}$ Millex-LG filter (Millipore, USA). The filtered supernatant was concentrated using a centrifugal concentrator (VC36S, TAITEC, Japan) for $2 \mathrm{~h}$. Identical samples were combined and subsequently freeze dried.

\subsection{Absolute quantification of intracellular metabolites}

The intracellular concentration of targeted metabolites namely acetyl-CoA, malonyl-CoA, butanoyl-CoA and pyruvate was quantified using ${ }^{13} \mathrm{C}$-labeled cell extract as internal standard (Wu et al. 2005; Dempo et al. 2014; Bennett et al. 2008). To prepare the cell extracts, wild type and BUOH-SE strains were cultured in $20 \mathrm{~mL}$ of BG-11 containing $50 \mathrm{mM} \mathrm{NaH}{ }^{13} \mathrm{CO}_{3}\left(>98\right.$ at. $\%{ }^{13} \mathrm{C}$, Isotec Inc., USA) for 2 days. Subsequently, the cells were inoculated in $100 \mathrm{~mL}$ of BG-11 containing $50 \mathrm{mM} \mathrm{NaH}{ }^{13} \mathrm{CO}_{3}$ at an initial $\mathrm{OD}_{730}$ of 0.04 . For medium feeding, BG-11 with 
$500 \mathrm{mM} \mathrm{NaH}{ }^{13} \mathrm{CO}_{3}$ was added into the culture. After 3 days of IPTG induction, cells were harvested by fast filtration using $1 \mu \mathrm{m}$ pore size Omnipore filter disks (Millipore, USA) and subsequently washed with pre-cooled deionized water. The intracellular metabolites extraction was performed using the method described above and repeated four times excluding the freeze drying step. After centrifugal concentration for $2 \mathrm{~h}$, all the samples were pooled in a $15 \mathrm{~mL}$ centrifuge tube. This pooled extract was used as an internal standard. The calibration curves for each metabolite were constructed according to Dempo et al. (2014) with minor modifications. Predetermined amounts of unlabeled standard mixtures were added into $2 \mathrm{~mL}$ of $80 \%$ methanol with ${ }^{13} \mathrm{C}$-labelled internal standard and extracted using the same procedure as described above. The peak area ratios of $\mathrm{U}_{-}{ }^{12} \mathrm{C}$ to $\mathrm{U}_{-}{ }^{13} \mathrm{C}$ metabolites were calculated using the LabSolutions software (Shimadzu Co., Japan) and plotted against their corresponding predetermined amounts of authentic standards. The calibration curves of targeted metabolites are listed in Table S1.

\section{$2.5{ }^{13} \mathrm{C}$-labelling experiment}

For metabolic turnover analysis, ${ }^{13} \mathrm{C}$-labelling experiment was carried out according to Hasunuma et al. (2013) with minor modifications. Cells pre-cultivated for 3 days after IPTG induction using the conditions mentioned above were filtered and resuspended in $50 \mathrm{~mL}$ of BG-11 with $25 \mathrm{mM}$ $\mathrm{NaH}^{13} \mathrm{CO}_{3}$. Four $\mathrm{mL}$ of culture broth was filtered at each time point $(0.5,1,2,5,10$ and $30 \mathrm{~min})$ and the cells that were retained on the filter papers were processed as described in the previous section. Based on Hasunuma et al. (2010), the ${ }^{13} \mathrm{C}$ fraction was calculated using the equation:

$m_{i}(\%)=\frac{M_{i}}{\sum_{j=0}^{n} M_{j}} \times 100$

${ }^{13} \mathrm{C}$ fraction $(\%)=\sum_{i=1}^{n} \frac{i \times m_{i}}{n}$

where $M_{i}$ represents the peak area for a given metabolite with $i{ }^{13} \mathrm{C}$ atoms, $m_{i}$ represents relative peak area for each isotopomer and $n$ represents the number of carbon atoms in the metabolite. Metabolic turnover rate of each metabolite was calculated from the initial slope of the ${ }^{13} \mathrm{C}$ fraction change with respect to time $(0.5-5 \mathrm{~min})$.

\subsection{IP-LC/QqQ-MS analysis}

The freeze-dried samples were dissolved in $30 \mu \mathrm{L}$ of ultrapure water for IP-LC/QqQ-MS analysis using a Shimadzu Nexera UHPLC system coupled with LCMS 8030 plus (Shimadzu Co., Japan). The protocol for LC/QqQ-MS analysis was based on Dempo et al. (2014) using an
L-column 2 ODS $(150 \mathrm{~mm} \times 2.1 \mathrm{~mm}, 3 \mu \mathrm{m}$, Chemicals Evaluation and Research Institute, Japan). The mobile phase A used was $10 \mathrm{mM}$ tributylamine and $15 \mathrm{mM}$ acetic acid in water, while mobile phase B was methanol. The flow rate was $0.2 \mathrm{~mL} / \mathrm{min}$ and column oven temperature was $40{ }^{\circ} \mathrm{C}$. Gradient curves were as follows: for acyl-CoAs, $0 \% \mathrm{~B}$ at 0 , $50 \% \mathrm{~B}$ at $1,60 \% \mathrm{~B}$ at $10,90 \% \mathrm{~B}$ at $11-13,0 \% \mathrm{~B}$ at 14-20 min, and for pyruvate, $0 \% \mathrm{~B}$ at $0,15 \% \mathrm{~B}$ at $2,25 \% \mathrm{~B}$ at $7,50 \% \mathrm{~B}$ at $9,100 \% \mathrm{~B}$ at $11-13,0 \% \mathrm{~B}$ at $13.01-18 \mathrm{~min}$. The analysis mode was negative ion mode. Probe position was $+1.5 \mathrm{~mm}$, desolvation line temperature was $250{ }^{\circ} \mathrm{C}$, nebulizer gas flow was $2 \mathrm{~L} / \mathrm{min}$, drying gas flow was 15 $\mathrm{L} / \mathrm{min}$, and heat block temperature was $400{ }^{\circ} \mathrm{C}$. The IP-LC/ QqQ-MS analysis was performed with multiple reaction monitoring (MRM). Analytical parameters for the quantitative analysis and turnover analysis are listed in Table S2.

\subsection{Determination of intracellular NADH and $\mathrm{NAD}^{+}$concentration}

Fluorescent NAD/NADH detection kit (Cell Technology, USA) was used for quantification of intracellular NADH and $\mathrm{NAD}^{+}$concentration following the manufacturer's protocol. For each extraction, $1 \mathrm{~mL}$ of culture broth (3 days after IPTG induction) was collected in a $1.5 \mathrm{~mL}$ microfuge tube and subsequently centrifuged at $16,000 \times g$ for $1 \mathrm{~min}$ at $4{ }^{\circ} \mathrm{C}$. After removal of the supernatant, the resulting cell pellet was immediately immersed in liquid nitrogen. Next, the pellet was resuspended in $100 \mu \mathrm{L}$ of the $\mathrm{NAD}^{+}$or $\mathrm{NAD}(\mathrm{H})$ extraction buffer and $100 \mu \mathrm{L}$ of the NAD/NADH lysis buffer. After the samples were heated at $60{ }^{\circ} \mathrm{C}$ for $20 \mathrm{~min}, 100 \mu \mathrm{L}$ of the reaction buffer and $200 \mu \mathrm{L}$ of the opposite $(\mathrm{NAD}(\mathrm{H})$ or $\mathrm{NAD}^{+}$) extraction buffer were added into the tube. The lysate was mixed and centrifuged at $8,000 \mathrm{~g}$ for $5 \mathrm{~min}$ at $4{ }^{\circ} \mathrm{C}$. The supernatant was then retrieved for $\mathrm{NAD}^{+}$and NADH analysis. The supernatant was added with the enzyme mix and fluorescent NADH reaction reagent according to the manufacturer's instructions. The mixture was incubated at room temperature under a dark condition for $90 \mathrm{~min}$. Readings were taken using an ARVO MX 1420 multilabel counter (PerkinElmer) with excitation at $544 \mathrm{~nm}$ and emission at $590 \mathrm{~nm}$.

\section{Results}

\subsection{Comparison of two pathways for the formation of acetoacetyl-CoA from acetyl-CoA using targeted CoA profiling method}

Formation of acetoacetyl-CoA from acetyl-CoA in CoAdependent 1-butanol biosynthesis can be achieved via two pathways. One is via the direct condensation of 
bimolecular acetyl-CoA catalyzed by thiolase ( $a t o B$-encoded enzyme) present in strain EL14 (Fig. 1; Table 1). This condensation reaction is thermodynamically unfavorable since it requires an acetyl-CoA pool to drive the reaction forward, which was thought to be unsuitable for the effective conversion of acetyl-CoA to acetoacetyl-CoA under photosynthetic condition (Lan and Liao 2011). The other is an ATP-driven malonyl-CoA-mediated reaction catalyzed by acetyl-CoA carboxylase and acetoacetyl-CoA synthase ( $a c c A B C D$ and nphT7-encoded enzyme, respectively) present in strains EL20, EL22 and BUOH-SE (Fig. 1; Table 1). ATP, serving as an alternative driving force, enabled 1-butanol biosynthesis under photosynthetic condition (Lan and Liao 2012). To further optimize the pathway, it is important to validate the proposed ATPdependent function at the metabolite level. To address this, we performed quantitative metabolic profiling of acylCoAs in the 1-butanol biosynthesis pathway. Compared to the wild type, the intracellular concentration of acetyl-CoA in strain EL14 decreased by 1.8 -fold (Fig. 2) while the concentration of malonyl-CoA did not change. In addition, butanoyl-CoA was also detected as could be seen in Fig. 2. As for strain EL20, there was a 37-fold decrease in the concentration of acetyl-CoA (Fig. 2), and 5.3-fold decrease for malonyl-CoA (Fig. 2) compared to the wild type. In addition, EL20 showed 2.1-fold increase in butanoyl-CoA compared to EL14, (Fig. 2). These data signify that strains EL14 and EL20 consumed acetyl-CoA to form butanoylCoA. Our result indicates that strain EL20 also consumed malonyl-CoA to form butanoyl-CoA. Moreover, strain EL20 more effectively converted acetyl-CoA to butanoylCoA than strain EL14 (Fig. 2). Hence, the ATP-driven malonyl-CoA-mediated reaction (present in EL 20 and

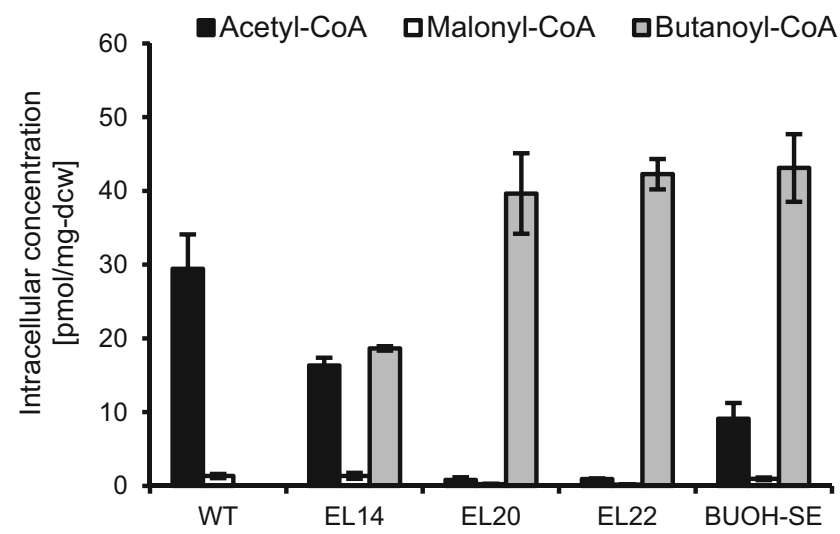

Fig. 2 Intracellular concentration of targeted acyl-CoAs namely acetyl-CoA, malonyl-CoA and butanoyl-CoA in strain wild type, EL14, EL20, EL22 and BUOH-SE. The concentration is described using $\mathrm{pmol} / \mathrm{mg}$-dry cell weight $(\mathrm{dcw})$ as a unit. Error bar shows standard deviation. 1-butanol concentration of all strains at 3 days after IPTG induction was lower than the LLOQ of GC-FID (lower limit of quantification: $5 \mathrm{mg} / \mathrm{L}$ ), with the exception of BUOH-SE strain $(86 \mathrm{mg} / \mathrm{L})$

BUOH-SE) facilitated the consumption of acetyl-CoA for 1-butanol biosynthesis better than the direct condensation reaction. More importantly, we were able to validate our CoA profiling method since the genotypes of the strains can be explained based on their differences at the metabolite level.

\subsection{Reductive reaction of butanoyl-CoA to butanal is a possible rate-limiting step of 1-butanol production in cyanobacteria}

NADPH is a more suitable driving force for the reductive reaction than NADH since the light reaction of

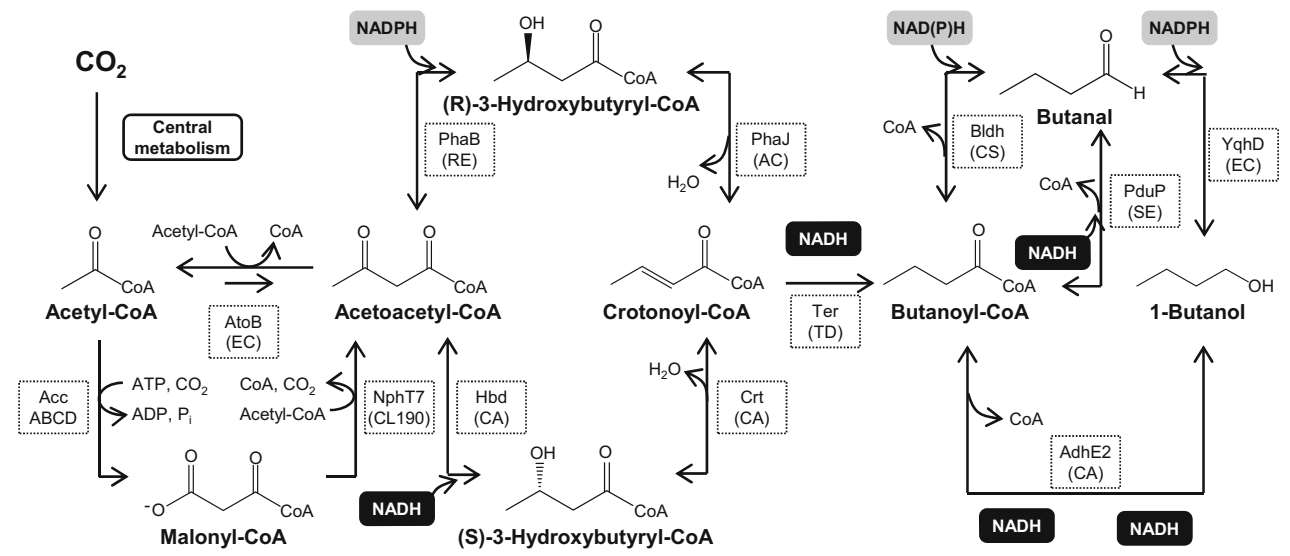

Fig. 1 Schematic representation of CoA-dependent 1-butanol biosynthesis pathway. Abbreviations: $A c c A B C D$ acetyl-CoA carboxylase, NphT7 acetoacetyl-CoA synthase, AtoB acetyl-CoA acetyltransferase, $\mathrm{Hbd}$ 3-hydroxybutyryl-CoA dehydrogenase, $\mathrm{Crt}$ crotonase, $P h a B$ acetoacetyl-CoA reductase, PhaJ $(R)$-specific crotonase, Ter trans- enoyl-CoA reductase, AdhE2 bifunctional aldehyde/alcohol dehydrogenase, Bldh CoA-acylating butanal dehydrogenase, PduP
CoA-acylating propionaldehyde dehydrogenase, YqhD NADPH-dependent alcohol dehydrogenase, EC Escherichia coli, CL190 Streptomyces sp. Strain CL190, CA Clostridium acetobutylicum, RE Ralstonia eutropha, AC Aeromonas caviae, TC Treponema denticola, CS Clostridium saccharoperbutylacetonicum N1-4, SE Salmonella enterica 
photosynthesis produces NADPH, resulting in a more abundant amount of NADPH than NADH in the cyanobacterial cell (Tamoi et al. 2005). Oxygen-tolerant enzymes are also preferable than oxygen-sensitive enzymes for cyanobacterial 1-butanol production due to oxygen evolution via a photosynthetic activity. Specifically, substituting the NADPH-dependent enzyme for an $\mathrm{NADH}$-dependent type in the reductive reaction of butanoyl-CoA to butanal played a key role in the improvement of 1-butanol productivity (Lan and Liao 2012). Furthermore, replacing the oxygen-sensitive enzyme with an oxygen-tolerant type dramatically increased 1-butanol productivity (Lan et al. 2013). In this study, strain EL20 possesses an oxygen-sensitive NADH-dependent aldehyde/ alcochol dehydrogenase encoded by adhE2, EL22 has the oxygen-sensitive $\mathrm{NAD}(\mathrm{P}) \mathrm{H}$-dependent butanal dehydrogenase encoded by bldH and BUOH-SE has the oxygentolerant NADH-dependent CoA-acylating propionaldehyde dehydrogenase encoded by $p d u P$ (Fig. 1; 1). We investigated the effect of each modification on the metabolic state of the pathway through quantitative metabolic profiling. Our result showed that the intracellular concentration of butanoyl-CoA in strain BUOH-SE was comparable to that of strains EL20 and EL22 and there was no significant difference in the intracellular butanoyl-CoA concentration between strains EL20, EL22 and BUOH-SE even though 1-butanol productivity of the strains was completely different (Table 1). These results indicate that there is still intracellular butanoyl-CoA available as a precursor for 1-butanol biosynthesis in spite of a series of improvements in the reductive reaction of butanoyl-CoA to butanal. Therefore, this suggests that butanoyl-CoA reduction is a possible limiting step for 1-butanol production. In this case, further enhancement of butanoyl-CoA reduction in cyanobacteria may be beneficial to increase 1-butanol productivity.

\subsection{Enhanced acetyl-CoA synthesis in strain BUOH- SE}

Intracellular acetyl-CoA and malonyl-CoA were depleted in strain EL22 due to the effective consumption of acetylCoA to butanoyl-CoA through malonyl-CoA as previously described (Fig. 2). However, there was an unexpected increase in the intracellular concentration of acetyl-CoA (9.8-fold) and malonyl-CoA (5.1-fold) in strain BUOH-SE compared to strain EL22 even though strain BUOH-SE also possesses an ATP-driven malonyl-CoA-mediated reaction (Fig. 2). Based on this observation, we hypothesized that the rate of acetyl-CoA and malonyl-CoA synthesis in strain BUOH-SE was increased compared to strain EL22 due to the effect of introducing the oxygen-tolerant enzyme PduP. In order to validate this hypothesis, we performed kinetic profiling of acetyl-CoA and butanoyl$\mathrm{CoA}$ by means of metabolic turnover analysis using ${ }^{13} \mathrm{C}$ labelled sodium bicarbonate. Kinetic profiling of malonylCoA was not done since the intracellular malonyl-CoA concentration of strain EL22 was too low to perform metabolic turnover analysis. Figure $3 \mathrm{a}$ shows the ${ }^{13} \mathrm{C}$ fraction change of acetyl-CoA with respect to time. The turnover rate of acetyl-CoA was estimated using the initial slope of the ${ }^{13} \mathrm{C}$ fraction versus time curve (Fig. 3c) in which unlabeled acetyl-CoA in strain BUOH-SE was replaced with ${ }^{13} \mathrm{C}$-labelled acetyl-CoA faster than in strain EL22 (at time point between 2 and $5 \mathrm{~min}$ ). The turnover rate of acetyl-CoA in strain BUOH-SE was higher than in strain EL22 (Fig. 3a, c). In this case, the intracellular concentration of acetyl-CoA was higher in strain $\mathrm{BUOH}-$ $\mathrm{SE}$ than in strain EL22 (Fig. 2). Since the rate of ${ }^{13} \mathrm{C}$ labeling depends on the pool size of each metabolite in the cell (Hasunuma et al. 2010), a given metabolite with higher intracellular concentration and turnover rate will have a higher carbon incorporation rate. Hence, the carbon incorporation rate of acetyl-CoA in strain BUOH-SE was increased compared to that of strain EL22 indicating that the rate of acetyl-CoA synthesis in strain BUOH-SE was higher than in strain EL22.

In case of butanoyl-CoA, a trend in the kinetic profiling similar to acetyl-CoA was also observed in which unlabeled butanoyl-CoA in strain BUOH-SE was replaced with ${ }^{13} \mathrm{C}$-labelled butanoyl-CoA faster than in strain EL22 (at time point between 2 and $10 \mathrm{~min}$ ). The turnover rate of butanoyl-CoA in strain BUOH-SE was also higher than in strain EL22 (Fig. 3b, c). Here, there was no significant difference in the intracellular concentration of butanoylCoA between strain EL22 and BUOH-SE (Fig. 2). Therefore, the increased carbon incorporation rate of butanoylCoA in strain BUOH-SE compared to strain EL22 indicated that the rate of butanoyl-CoA synthesis in strain BUOH-SE was higher than in strain EL22.

\subsection{Investigation of the factors that affect the increased rate of acetyl-CoA synthesis in strain BUOH-SE}

Next, we investigated the factors that contributed to the increased rate of acetyl-CoA synthesis in strain BUOH-SE. Pyruvate is a main precursor for acetyl-CoA biosynthesis through the central metabolism, thus the increased carbon incorporation rate of acetyl-CoA is expected to correlate with increased pyruvate consumption. Under the assumption that the effective regeneration of $\mathrm{NAD}^{+}$via the reductive reaction catalyzed by $\mathrm{PduP}$ enabled the increased supply of $\mathrm{NAD}^{+}$for the conversion of pyruvate to acetylCoA catalyzed by pyruvate dehydrogenase complex (PDH), we initially hypothesized that the insufficient 
Fig. $3{ }^{13} \mathrm{C}$ fraction change of acetyl-CoA (a) and butanoyl$\mathrm{CoA}(\mathbf{b})$ with respect to time in strain EL22 and BUOH-SE. c Estimated metabolic turnover rate of acetyl-CoA and butanoyl-CoA in strain EL22 and BUOH-SE. The turnover rate was calculated on the basis of the initial slope of ${ }^{13} \mathrm{C}$ fraction versus time plot. Asterisk (*) means a statistically-significant difference in turnover rate of the corresponding metabolite between the strains at $P<0.05$ by means of Student's $t$ test. Error bar shows standard deviation
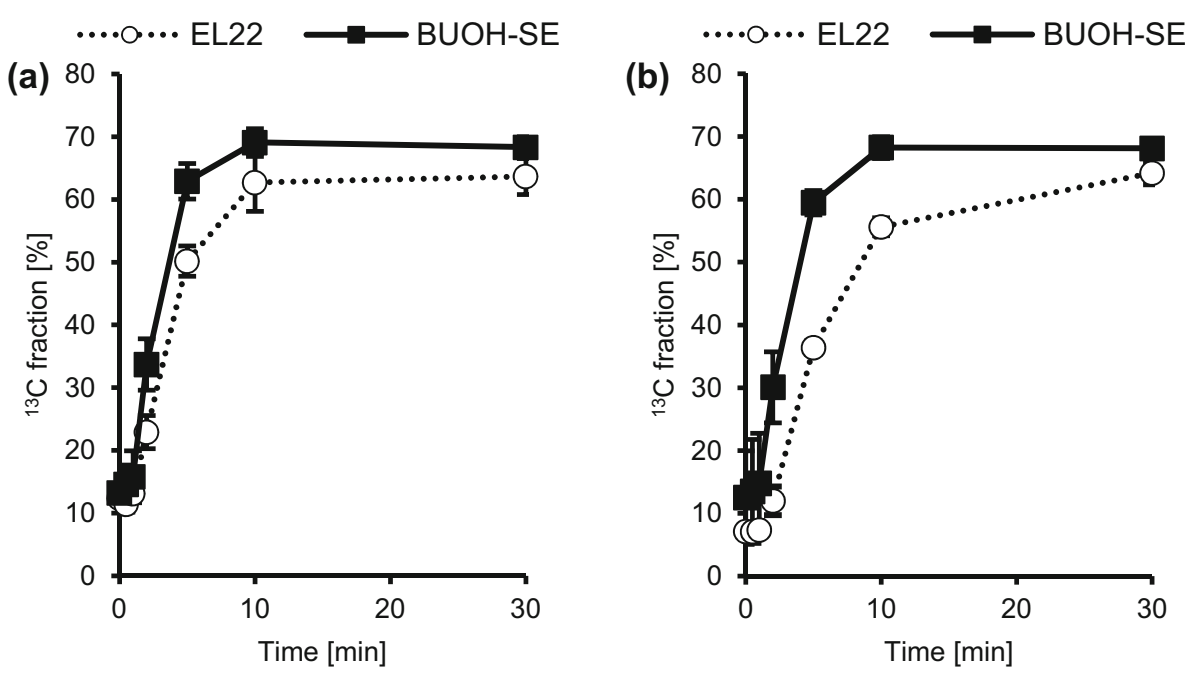

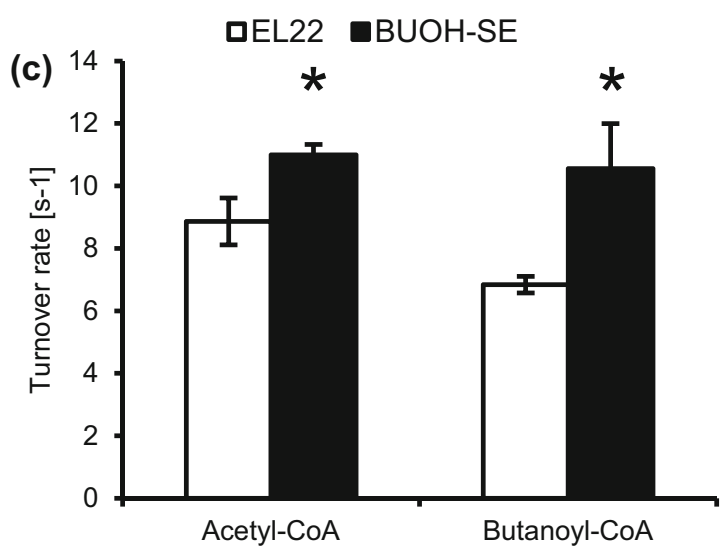

$\mathrm{NAD}^{+}$pool was the limiting factor of strain EL22 for converting pyruvate to acetyl-CoA via PDH. However, our result showed that the intracellular $\mathrm{NAD}^{+}$concentration of strain EL22 was slightly higher than that of strain BUOHSE (Table 2), which meant that the intracellular $\mathrm{NAD}^{+}$ concentration of strain EL22 is enough to convert pyruvate to acetyl-CoA via PDH and is therefore not the limiting factor of EL22.

Thus, we focused on free CoA, the co-factor that is necessary for the conversion of pyruvate to acetyl-CoA. We assumed that a more effective regeneration of free CoA from butanoyl-CoA via the reductive reaction catalyzed by PduP enabled the adequate supply of free CoA for the conversion of pyruvate to acetyl-CoA. Since free CoA is undetectable due to its low intracellular concentration, we constructed a strain BUOH-SE w/o pduP incapable of regenerating free $\mathrm{CoA}$ from butanoyl-CoA to investigate how free CoA that was regenerated from butanoyl-CoA affected the conversion of pyruvate to acetyl-CoA. In this strain, ter, nphT7, phaJ, and phaB genes were expressed but not PduP (Table 1) thus, it was identical to strain EL22 or BUOH-SE except for its ability to produce 1-butanol from butanoyl-CoA through butanal. Figure 4 shows the ratio of intracellular concentration of pyruvate to acetylCoA (PYR/AcCoA ratio) in wild type, strains EL22, BUOH-SE w/o $p d u P$, and BUOH-SE. PYR/AcCoA ratio of strain BUOH-SE markedly decreased compared to that of strain EL22, which indicated that the conversion of

Table 2 Intracellular concentration of $\mathrm{NAD}^{+}$and NADH and intracellular NADH/NAD ${ }^{+}$ratio in strain EL22 and BUOH-SE

\begin{tabular}{llll}
\hline & NAD $^{+}(\mathrm{pmol} / \mathrm{mg}-\mathrm{dcw})$ & $\mathrm{NADH}(\mathrm{pmol} / \mathrm{mg}-\mathrm{dcw})$ & $\mathrm{NADH} \mathrm{NAD}^{+}$ratio \\
\hline EL22 & $161 \pm 23$ & $44 \pm 20$ & $0.29 \pm 0.13$ \\
BUOH-SE & $135 \pm 13$ & $51 \pm 16$ & $0.39 \pm 0.14$ \\
\hline
\end{tabular}

The values are the mean $\pm \mathrm{SD}$ of three replicates 
pyruvate to acetyl-CoA was enhanced in strain BUOH-SE compared to strain EL22. In contrast, strain BUOH-SE w/o pduP showed a higher PYR/AcCoA ratio than EL22 (Fig. 4), which strongly suggests that free CoA has to be regenerated from butanoyl-CoA for the conversion of pyruvate to acetyl-CoA. In case of strain BUOH-SE, the introduction of PduP gene unexpectedly enhanced free CoA recycling, resulting to an increase in 1-butanol production.

\section{Discussion}

In this study, we characterized the differences in 1-butanol biosynthesis of strains with different genetic backgrounds by quantitative metabolic profiling of acyl-CoAs. Acetoacetyl-CoA, 3-hydroxybutyryl-CoA and crotonoyl-CoA were undetectable in all strains, and as well as their corresponding ${ }^{13} \mathrm{C}$-labelled forms in the ${ }^{13} \mathrm{C}$-labelled cell extract. Considering the fact that there was no significant loss of the metabolites during the extraction procedure (Table S3), we inferred that the intracellular concentration of these three acyl-CoAs was too low compared to the detectable levels of other acyl-CoAs namely acetyl-CoA, malonyl-CoA and butanoyl-CoA. We therefore inferred that there was no bottleneck reaction in the conversion of acetoacetyl-CoA to crotonoyl-CoA through 3-hydroxybutyryl-CoA. In fact, acetyl-CoA was effectively converted to butanoyl-CoA in both NADH-dependent reaction (in strain EL14 and strain EL20) and NADPH-dependent reaction (in strain EL22 and strain BUOH-SE) (Figs. 1, 2). In the case of low expression levels of (S)-3-hydroxybutyryl-CoA dehydrogenase encoded by $h b d$, the conversion of acetoacetyl-CoA to $(S)$-3-hydroxybutyryl-CoA becomes a rate-limiting step in the production of butanoyl-CoA (Fischer et al. 2010). Therefore, the expression levels of (S)-3hydroxybutyryl-CoA dehydrogenase in the 1-butanol-producing cyanobacteria may be enough to facilitate the

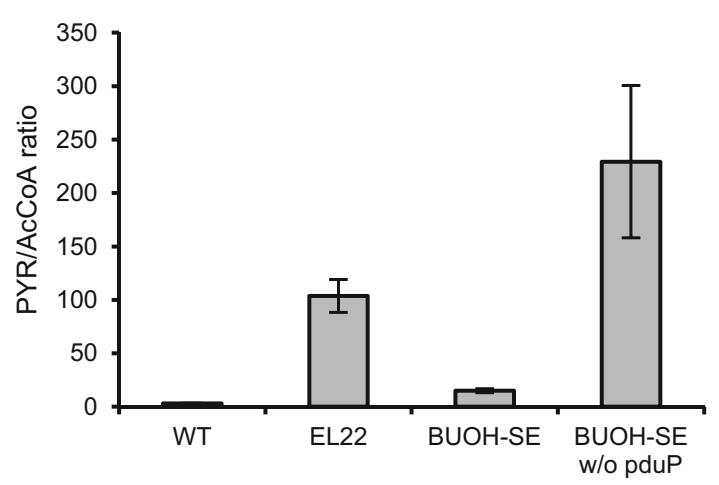

Fig. 4 PYR/AcCoA ratio in wild type, strain EL22, BUOH-SE w/o $p d u P$ and BUOH-SE. Error bar shows standard deviation conversion of acetoacetyl-CoA to $(S)$-3-hydroxybutyrylCoA. Regarding the conversion of crotonoyl-CoA to butanoyl-CoA, it is catalyzed by the NADH-dependent trans-enoyl-CoA reductase Ter derived from Treponema denticola in the engineered cyanobacteria. This enzyme is oxygen-tolerant and irreversible compared to the native enzyme in Clostridium species catalyzing the conversion of crotonoyl-CoA to butanoyl-CoA, which is oxygen-sensitive and reversible (Inui et al. 2008). Hence, it is possible that there was a more straightforward conversion of crotonoyl-CoA to butanoyl-CoA in the engineered cyanobacteria. Using quantitative target analysis of acyl-CoAs in the 1-butanol biosynthesis, we validated our previous hypothesis that the oxygen sensitivity of CoA-acylating aldehyde dehydrogenase is a possible key limiting factor in cyanobacteria for the production of 1-butanol through the CoA-dependent pathway (Lan et al. 2013). In this study, we also showed that intracellular butanoyl-CoA is still available as a precursor for 1-butanol biosynthesis. Therefore, improving the enzyme activity of CoA-acylating aldehyde dehydrogenase or substituting another enzyme with more preferable kinetic parameters such as $K_{\mathrm{m}}$ and $k_{\text {cat }}$ may further improve the 1-butanol productivity. Another presumable strategy is to utilize acetate CoAtransferase which catalyzes the reaction of butyrate and acetyl-CoA production from butanoyl-CoA and acetate (Vanderwinkel et al. 1968). Subsequently, butyrate can be converted to 1-butanol via two reactions catalyzed by carboxylic acid reductase and aldehyde dehydrogenase, respectively (Pásztor et al. 2014). This additional bypassing pathway may play a role to increase consumption rate of butanoyl-CoA for 1-butanol biosynthesis.

Using metabolic turnover analysis, kinetic profiling also revealed the unexpected effect of introducing the oxygentolerant PduP for enhancing acetyl-CoA synthesis. Considering the higher $\mathrm{PYR} / \mathrm{AcC} \mathrm{A}$ ratio in strain EL22 compared to wild type (Fig. 4), the conversion of pyruvate to acetyl-CoA is a possible bottleneck step in strain EL22 for 1-butanol biosynthesis through the CoA-dependent pathway. The increased rate of acetyl-CoA synthesis and the decreased PYR/AcCoA ratio in strain BUOH-SE compared to strain EL22, however, indicate that strain BUOH-SE overcame the bottleneck step. Intracellular $\mathrm{NAD}^{+}$and NADH pools were comparable in strain EL22 and BUOH-SE (Table 2), thus the redox co-factor regeneration was not a limiting factor for the conversion of pyruvate to acetyl-CoA via PDH. Strain BUOH-SE w/o $p d u P$ is incapable of regenerating free-CoA from butanoylCoA because it lacks CoA-acylating aldehyde dehydrogenase. Since the PYR/AcCoA ratio of the strain BUOH-SE w/o $p d u P$ was also high similar with strain EL22 (Fig. 4), we therefore infer that regeneration of free $\mathrm{CoA}$ from butanoyl-CoA is a key factor for the conversion of 
pyruvate to acetyl-CoA. It has been reported that in vitro synthetic system for the poly (3-hydroxybutyric acid) formation can utilize free CoA recycling (Jossek and Steinbüchel 1998) and repeated recycling of free CoA in rat liver mitochondria occurs (Osmundsen and Sherratt 1975). Therefore, it is reasonable to assume that free CoA released from butanoyl-CoA is recycled for the conversion of pyruvate to acetyl-CoA in the cyanobacteria. We expected that butanoyl-CoA would accumulate in strain BUOH-SE w/o $p d u P$ more than strain EL22 and BUOH-SE, however, the intracellular concentration was comparable among the strains (Fig. 4). Moreover, the sum of the intracellular concentration of acetyl-CoA, malonyl-CoA and butanoylCoA was also comparable among the strains (Fig. 4). These results indicate that the amount of the intracellular acyl-CoAs available in the CoA-dependent 1-butanol biosynthesis pathway has an upper limit. This fact also indicates the importance of regeneration of free-CoA from butanoyl-CoA for the further synthesis of acetyl-CoA from pyruvate. Considering such importance of regeneration of free $\mathrm{CoA}$ from butanoyl-CoA for increasing the rate of acetyl-CoA synthesis, the reduction of butanoyl-CoA to butanal must be enhanced to improve the recycling efficiency of free-CoA.

\section{Conclusion}

We investigated in vivo the metabolic state in the CoAdependent 1-butanol biosynthesis pathway of cyanobacteria by quantitative target analysis and kinetic profiling of acyl-CoAs in the CoA-dependent pathway through (RP-IPLC)/QqQ-MS. The quantification of intracellular concentration of acyl-CoAs in the 1-butanol biosynthesis pathway validated at the metabolite level that the ATP-driven malonyl-CoA-mediated reaction effectively consumed acetyl-CoA for butanoyl-CoA synthesis as intended and indicated that the reductive reaction of butanoyl-CoA to butanal was a possible rate-limiting step in the cyanobacterial 1-butanol biosynthesis through the CoA-dependent pathway. Moreover, the kinetic profiling of acetyl-CoA and butanoyl-CoA using metabolic turnover analysis revealed the unexpected increase of acetyl-CoA synthesis rate in strain BUOH-SE. We inferred that the effective regeneration of free-CoA from butanoyl-CoA played an important role in enhancing the conversion of pyruvate to acetylCoA. These facts strongly indicate that the reductive reaction of butanoyl-CoA to butanal should be additionally modified to improve 1-butanol productivity in the cyanobacteria. The information shown in this study should be helpful for further engineering of cyanobacteria for improved 1-butanol production.
Acknowledgments This work was supported by Japan Science and Technology Agency (JST) and National Science Foundation (NSF) under "JST-NSF: Metabolomics for Low Carbon Society" project. We would like to thank Hiroshi Shimizu and Katsunori Yoshikawa for their assistance in the $\mathrm{NADH}$ and $\mathrm{NAD}^{+}$assay.

\section{Compliance with ethical standards}

Conflict of Interest We have no conflict of interest to declare for conduct of this study and/or preparation of this manuscript.

Human and animal rights statements This article does not contain any studies with human participants or animals performed by any of the authors.

Informed consent Informed consent was obtained from all individual participants included in the study

Open Access This article is distributed under the terms of the Creative Commons Attribution 4.0 International License (http://creative commons.org/licenses/by/4.0/), which permits unrestricted use, distribution, and reproduction in any medium, provided you give appropriate credit to the original author(s) and the source, provide a link to the Creative Commons license, and indicate if changes were made.

\section{References}

Atsumi, S., Higashide, W., \& Liao, J. C. (2009). Direct photosynthetic recycling of carbon dioxide to isobutyraldehyde. Nature Biotechnology, 27, 1177-1180.

Bennett, B. D., Yuan, J., Kimball, E. H., \& Rabinowitz, J. D. (2008). Absolute quantification of intracellular metabolite concentrations by an isotope ratio-based approach. Nature Protocols, 3, 1299-1311.

Dempo, Y., Ohta, E., Nakayama, Y., Bamba, T., \& Fukusaki, E. (2014). Molar-based targeted metabolic profiling of cyanobacterial strains with potential for biological production. Metabolites, 4, 499-516.

Deng, M., \& Coleman, J. R. (1999). Ethanol synthesis by genetic engineering in cyanobacteria. Applied and Environmental Microbiology, 65, 523-528.

Dexter, J., \& Fu, Pe. (2009). Metabolic engineering of cyanobacteria for ethanol production. Energy \& Environmental Science, 2, $857-864$

Dürre, P. (2007). Biobutanol: An attractive biofuel. Biotechnology Journal, 2, 1525-1534.

Fischer, C. R., Tseng, H. C., Tai, M., Prather, K. L. J., \& Stephanopoulos, G. (2010). Assessment of heterologous butyrate and butanol pathway activity by measurement of intracellular pathway intermediates in recombinant Escherichia coli. Applied Microbial and Cell Physiology, 88, 265-275.

Gao, Z., Zhao, H., Li, Z., Tana, X., \& Lu, X. (2012). Photosynthetic production of ethanol from carbon dioxide in genetically engineered cyanobacteria. Energy \& Environmental Science, 5, 9857-9865.

Gheshlaghi, R., Scharer, J. M., Moo-Young, M., \& Chou, C. P. (2009). Metabolic pathways of clostridia for producing butanol. Biotechnology Advances, 6, 764-781.

Gibson, D. G., Young, L., Chuang, R. Y., Venter, J. C., Hutchison, C. A, I. I. I., \& Smith, H. O. (2009). Enzymatic assembly of DNA molecules up to several hundred kilobases. Nature Methods, 6 , $12-16$.

Hasunuma, T., Harada, K., Miyazawa, S., Kondo, A., Fukusaki, E., \& Miyake, C. (2010). Metabolic turnover analysis by a 
combination of in vivo ${ }^{13} \mathrm{C}$-labelling from ${ }^{13} \mathrm{CO}_{2}$ and metabolic profiling with CE-MS/MS reveals rate-limiting steps of the $\mathrm{C}_{3}$ photosynthetic pathway in Nicotiana tabacum leaves. Journal of Experimental Botany, 61, 1041-1051.

Hasunuma, T., Kikuyama, F., Matsuda, M., Aikawa, S., Izumi, Y., \& Kondo, A. (2013). Dynamic metabolic profiling of cyanobacterial glycogen biosynthesis under conditions of nitrate depletion. Journal of Experimental Botany, 64, 2943-2954.

Inui, M., Suda, M., Kimura, S., et al. (2008). Expression of Clostridium acetobutylicum butanol synthetic genes in Escherichia coli. Applied Genetics and Molecular Biotechnology, 77, 1305-1316.

Jin, H., Chen, L., Wang, J., \& Zhang, W. (2014). Engineering biofuel tolerance in non-native producing microorganisms. Biotechnology Advances, 32, 541-548.

Jossek, R., \& Steinbüchel, A. (1998). In vitro synthesis of poly(3hydroxybutyric acid) by using an enzymatic coenzyme A recycling system. Federation of European Microbiological Societies Microbiology Letters, 168, 319-324.

Lan, E. I., \& Liao, J. C. (2011). Metabolic engineering of cyanobacteria for 1-butanol production from carbon dioxide. Metabolic Engineering, 13, 353-363.

Lan, E. I., \& Liao, J. C. (2012). ATP drives direct photosynthetic production of 1-butanol in cyanobacteria. Proceedings of the National Academy of Sciences of the United States of America, 109, 6018-6023.

Lan, E. I., Ro, S. Y., \& Liao, J. C. (2013). Oxygen-tolerant coenzyme A-acylating aldehyde dehydrogenase facilitates efficient photosynthetic n-butanol biosynthesis in cyanobacteria. Energy \& Environmental Science, 6, 2672-2681.

Lindberg, P., Park, S., \& Melis, A. (2010). Engineering a platform for photosynthetic isoprene production in cyanobacteria, using Synechocystis as the model organism. Metabolic Engineering, 12, 70-79.

Liua, X., Shengb, J., \& Curtiss, R, I. I. I. (2011). Fatty acid production in genetically modified cyanobacteria. Proceedings of the National Academy of Sciences of the United States of America, 108, 6899-6904.
Machado, I. M. P., \& Atsumi, S. (2012). Cyanobacterial biofuel production. Journal of Biotechnology, 162, 50-56.

Oliver, J. W. K., Machado, I. M. P., Yoneda, H., \& Atsumi, S. (2013). Cyanobacterial conversion of carbon dioxide to 2,3-butanediol. Proceedings of the National Academy of Sciences of the United States of America, 110, 1249-1254.

Osmundsen, H., \& Sherratt, H. S. A. (1975). A novel mechanism for inhibition of $\beta$-oxidation by methylenecyclopropylacetyl-CoA, a metabolite of hypoglycin. Federation of European Biochemical Societies Letters, 55, 1-4.

Pásztor, A., Kallio, P., Malatinszky, D., Akhtar, M. K., \& Jones, P. R. (2014). A synthetic $\mathrm{O}_{2}$-tolerant butanol pathway exploiting native fatty acid biosynthesis in Escherichia coli. Biotechnology and Bioengineering,. doi:10.1002/bit.25324.

Shen, C. R., Lan, E. I., Dekishima, Y., Baez, A., Cho, K. M., \& Liao, J. C. (2011). Driving forces enable high-titer anaerobic 1-butanol synthesis in Escherichia coli. Applied and Environmental Microbiology, 77, 2905-2915.

Tamoi, M., Miyazaki, T., Fukamizo, T., \& Shigeoka, S. (2005). The Calvin cycle in cyanobacteria is regulated by $\mathrm{CP} 12$ via the $\mathrm{NAD}(\mathrm{H}) / \mathrm{NADP}(\mathrm{H})$ ratio under light/dark conditions. The Plant Journal, 42, 504-513.

Vanderwinkel, E., Furmanski, P., Reeves, H. C., \& Ajl, S. J. (1968). Growth of Escherichia coli on fatty acids: Requirement for coenzyme A transferase activity. Biochemical and Biophysical Research Communications, 33, 902-908.

Varman, A. M., Xiao, Y., Pakrasi, H. B., \& Tang, Y. J. (2012). Metabolic engineering of Synechocystis sp. strain PCC 6803 for isobutanol production. Applied and Environmental Microbiology, 79, 908-914

Wang, W., Liu, X., \& Lu, X. (2013). Engineering cyanobacteria to improve photosynthetic production of alka(e)nes. Biotechnology for Biofuels, 6, 69-78.

Wu, L., Mashego, M. R., Van Dam, J. C., et al. (2005). Quantitative analysis of the microbial metabolome by isotope dilution mass spectrometry using uniformly ${ }^{13} \mathrm{C}$-labeled cell extracts as internal standards. Analytical Biochemistry, 336, 164-171. 